\title{
A NEW METHOD FOR DETERMINING THE HELIUM ABUNDANCE IN THE SOLAR ATMOSPHERE*
}

\author{
(Invited Review) \\ G. R. ISAA K
}

Department of Physics, University of Birmingham, P.0. Box 363, Birmingham, B15 2TT, U.K.

\begin{abstract}
Recent observations of a cut-off frequency in the acoustic modes of the Sun (Claverie et al., 1981b) should help determine the mean molecular weight and, thereby, the helium abundance in the visible layers of the solar atmosphere. A first preliminary result of $Y=0.42 \pm 0.04$ is obtained for an assumed minimum photospheric temperature of $4400 \mathrm{~K} \pm 200 \mathrm{~K}$.
\end{abstract}

The determination of the helium abundance $Y$ (by weight) in the Sun's interior would help elucidate the physics and astrophysics of the solar neutrino problem (Iben, 1969) and would presumably provide a firm upper bound to the cosmological helium abundance as no means of destroying helium in the Sun is known. The observation (Claverie et al., 1979, 1980, 1981a; Grec et al., 1980) of the structure in the global oscillations with periods near $5 \mathrm{~min}$ and their interpretation (Claverie et al., 1979) in terms of two then current models (Iben and Mahaffy, 1976; Christensen-Dalsgaard et al., 1979) suggested a low $Z$ and low $Y$ (Isaak, 1980). This has been questioned by Christensen-Dalsgaard and Gough (1980) but the facts on which they based their criticism are suspect. These authors adopted a mean spacing between neighbouring modes of the same kind of $136.0 \mu \mathrm{Hz}$ compared with the observed value of $135.2 \pm 0.2 \mu \mathrm{Hz}$ obtained earlier and remeasured in 1980 (Claverie et al., 1981a). The cut off frequency of $5.7 \mu \mathrm{Hz}$ observed by (Claverie et al., 1981b) (see below) is substantially higher than the $5 \mathrm{mHz}$ these authors assumed. Consequently the model frequencies near $3 \mathrm{mHz}$ require corrections which are smaller than the authors claim.

It is, nevertheless, very likely that a clear picture will not emerge until lower order modes, particularly the fundamental radial mode and its overtones are observed (Brookes et al., 1976) and unambiguously identified.

The determination of the helium abundance in the solar atmosphere would form a substitute for measurements of $Y$ if one assumes that no segregation effects between the interior and the atmosphere occur. Even such measurements have been all but impossible (Unsöld, 1969).

The time-averaged composition of the solar wind (Hundhausen, 1972) is consistent with a $Y=0.16$ but it is conceivable that selection effects exist in the solar wind and possibly even in the solar photosphere.

* Proceedings of the 66th IAU Colloquium: Problems in Solar and Stellar Oscillations, held at the Crimean Astrophysical Observatory, U.S.S.R., 1-5 September, 1981. 
The aim of this note is to suggest a new method of determining the mean molecular weight of the upper photosphere and lower chromosphere, predominantly determined by hydrogen and helium abundances, and to report a first preliminary result using such a method.

It is well known (Bray and Loughhead, 1974) that an isothermal gravitationally stratified atmosphere has a Lamb acoustic cut off frequency $v_{1}=\gamma g / 4 \pi v$ for vertically propagating waves, where $\gamma$ is the ratio of specific heats, $g$ is the acceleration due to gravity, and $v$ is the local velocity of sound. The modifications due to radiative damping and the small magnetic field in the upper photosphere appear small (Bray and Loughhead, 1974) so long as one observes the cut-off frequency near the temperature minimum, thereby also approximating to the isothermal condition.

Recently it was discovered (Claverie et al., 1981b) using the $7699 \AA$ resonance line of neutral potassium that the acoustic modes centred on $3 \mathrm{mHz}$ appear to have a cut-off frequency at $v_{C}=5.7 \mathrm{mHz}$.

According to de la Reza and Müller (1972) and Wiehr (1981), the part of the spectral line used in the velocity spectroscopy by the Birmingham group lies between about $\log \tau=-2$ and $-1.6, \tau$ being the optical depth. Below this frequency $v_{C}$ considerable power was seen in the mean velocity spectrum (averaged over 8 days) whereas above $v_{C}$ the power level is constant and corresponds to a velocity amplitude of $1.2 \mathrm{~cm} \mathrm{~s}^{-1}$ r.m.s. per channel.

This level appears to be due to finite photon statistics, instrumental noise and any residual noise due to the terrestrial atmosphere. This frequency $v_{C}$ was identified with the Lamb cut-off frequency $v_{1}$.

The mean molecular weight $m$ can be estimated from $v_{C}, g$ and the velocity of sound given by $v=(\gamma k T / m)^{1 / 2}, k$ being Boltzmann's constant, and $T$ the absolute temperature. $\gamma$ and $T$ can be estimated from a model of the visible layers of the solar atmosphere.

Although $\gamma=\frac{5}{3}$ to a good approximation (Ulmschneider, 1967), estimates of the temperature, near the temperature minimum, seem to range from $4600 \mathrm{~K}$ to $4200 \mathrm{~K}$.

The resultant mean molecular weight ranges from 1.57 to 1.43 , with corresponding $Y$ values of 0.46 and 0.38 assuming $Z=0.02$ and single ionization for $\mathrm{C}, \mathrm{N}, \mathrm{O}$, etc. and no appreciable ionization for hydrogen and helium.

Thus for an adopted mean temperature of $4400 \mathrm{~K} \pm 200 \mathrm{~K}$ the resultant helium abundance is found to be $Y=0.42 \pm 0.04$.

It is interesting to point out that this is consistent with the result $Y=0.38$ obtained by Unsöld (1969).

It is conceivable that two dimensional velocity spectroscopy of the Sun will achieve a sufficient resolution and stability in the near future to measure the velocity of sound propagation directly. An explosive event (flare, meteorite, comet) will produce a detectable shock wave the progress of which will be followed. Such a measurement might provide yet another method of determining the mean molecular weight in the visible layers of the Sun. 


\section{References}

Bray, R. J. and Loughhead, R. E.: 1974, The Solar Chromosphere, Chapter 6, Chapman and Hall, London. Brookes, J. R., Isaak, G. R., and van der Raay, H. B.: 1976, Nature 259, 92.

Christensen-Dalsgaard, J., Gough, D. O., and Morgan, J. G.: 1979, Astron. Astrophys. 73, 121.

Christensen-Dalsgaard, J. and Gough, D. O.: 1980, Nature 288, 544.

Claverie, A., Isaak, G. R., McLeod, C. P., van der Raay, H. B., and Roca Cortes, T.: 1979, Nature 282, 591.

Claverie, A., Isaak, G. R., McLeod, C. P., van der Raay, H. B., and Roca Cortes, T.: 1980, Astron. Astrophys. 91, L9.

Claverie, A., Isaak, G. R., McLeod, C. P., van der Raay, H. B., and Roca Cortes, T.: 1981a, Nature 293, 443.

Claverie, A., Isaak, G. R., McLeod, C. P., van der Raay, H. B., and Roca Cortes, T.: 1981b, Solar Phys. $\mathbf{7 4}, 51$.

de la Reza, R. and Müller, E. A.: 1975, Solar Phys. 43, 15.

Grec, G., Fossat, E., Pomerantz, M.: 1980, Nature 288, 541.

Hundhausen, A. J.: 1972, Coronal Expansion and the Solar Wind, Springer, Berlin, p. 99.

Iben, I.: 1969, Ann. Phys. 54, 164.

Iben, I. and Mahaffy, J.: 1976, Astrophys. J. Letters 209, L39.

Isaak, G. R.: 1980, Nature 283, 644.

Ulmschneider, P.: 1967, Z. Astrophys. 67, 193.

Unsöld, A. O. J.: 1969, Science 163, 1015.

Wiehr, E.: 1981, private communication, Göttingen. 